exchange mechanism called "distributive conjugative transfer (DCT)," recently discovered in other mycobacterial species (Gray et al., Science. 2016. 354: 347-350). We also identified chromosomal loci where allelic variation was correlated with geographic origin. One noteworthy discovery was that the origin of genes responsible for trehalose biosynthesis differed between Asian and other MAH populations. Furthermore, we observed transmission of alleles encoding "mammalian cell entry proteins" between East Asian populations. Therefore, sexual reproduction, likely via DCT, plays a critical role in local adaptation of MAH.

As a new concept, we present a model for the life cycle of $M$. avium, in which $M$. avium generates progeny with diverse genomes via "mating" in a common environmental pool, prior to infection of human hosts. After infection, the progeny is subjected to natural selection within the host, followed by the re-release of clones with adaptive alleles into the environment. This concept may also be relevant for other mycobacterial species.

6.23 doi: 10.15789/2220-7619-2018-4-6.23

\section{UTILITY OF WHOLE GENOME SEQUENCING (WGS) OF MYCOBACTERIUM TUBERCULOSIS COMPLEX ISOLATES IN PRACTISE}

R. Jajou ${ }^{1}$, A. de Neeling ${ }^{1}$, S. Lipworth ${ }^{2}$, T. Walker ${ }^{2}$, R. Anthony ${ }^{1}$, D. van Soolingen ${ }^{1}$

${ }^{I}$ National Institute for Public Health and the Environment (RIVM), Bilthoven, The Netherlands; ${ }^{2}$ Nuffield Department of Medicine, University of Oxford, John Radcliffe Hospital, Oxford, United Kingdom

Since 2016, all culture positive M. tuberculosis complex isolates have been subjected to WGS to allow comparison with the current laboratory diagnosis in the Netherlands. The utility of WGS was investigated for 1) identification of (sub) species and genotypes; 2) drug susceptibility testing; and 3) investigation on tuberculosis transmission.

The new SNP-IT (SNPs to identify TB) method, developed at the RIVM/the Netherlands and Oxford University/ the UK, traces SNPs shared exclusively by members of each (sub) species, lineage, and sub-lineage. The total number of unique SNPs identified ranged from 23 for $M$. bovis to 6.837 for $M$. canettii. The SNP-IT method was applied to 1.157 routine samples from 2016/2017 in the Netherlands and compared with identification by Reverse Line Blot, PhyResSe, and Coll SNP-barcode. This comparison showed that SNP-IT more accurately identifies all animal (sub) species and is more specific in identifying sub-lineages of lineage 4. A small proportion $(n=176)$ of lineage 4 isolates could not be identified by SNP-IT due to high similarity to the H37Rv reference genome; these are in the Coll SNP-barcode system identified as lineage 4.5/4.7/4.8.

Phenotypic drug susceptibility testing (MGIT) was compared with the detection of resistance-associated mutations by WGS for first-line antibiotics rifampicin, isoniazid, ethambutol, and pyrazinamide. In total, 1.134 isolates from 2016/2017 in the Netherlands were included. For all drugs, the negative predictive value (NPV) was $>99 \%$. In general, rifampicin and isoniazid had most optimal scores. For rifampicin, the sensitivity was $100 \%$, specificity $99.8 \%$, the positive predictive value $95 \%$, and the NPV $100 \%$. This was $98 \%, 99.2 \%, 92.5 \%$, and $99.8 \%$, respectively, for isoniazid. WGS was also able to predict intermediate/low level resistance for rifampicin, isoniazid, and pyrazinamide. A minority of isolates showed discrepancy between MGIT and WGS results; these isolates are re-tested to explain discrepancy results.
Both VNTR typing and WGS were applied to all isolates from 2016. In total, 535 isolates were genotyped, of which $25 \%(134 / 535)$ were clustered by VNTR and $15 \%$ $(82 / 535)$ by WGS. The proportion of identified epi-links among WGS clustered cases $(50 \%)$ was much higher than among VNTR clustered cases $(31 \%)$. This study was repeated with isolates from 2016 and 2017 to analyse transmission over two years.

\section{MINOR GENETIC DETERMINANTS OF SECOND-LINE INJECTION DRUGS RESISTANCE IN MYCOBACTERIUM TUBERCULOSIS}

R. Jou ${ }^{1,2,3}$, E.V. Kulagina ${ }^{4}$, W.T. Lee ${ }^{1,2}$, E.Yu. Nosova ${ }^{5}$, J.Y. Weng ${ }^{1,2}$, O.V. Antonova ${ }^{4}$, W.H. Lin ${ }^{1,2}$, A.I. Isakova ${ }^{5}$, M.H. Wu ${ }^{1,2}$, D.V. Zimenkov ${ }^{4}$

${ }^{1}$ Tuberculosis Research Center, Taiwan Centers for Disease Control, Taipei, Taiwan; ${ }^{2}$ Diagnostics and Vaccine Center, Taiwan Centers for Disease Control, Taipei, Taiwan; ${ }^{3}$ Institute of Microbiology and Immunology, National Yang-Ming University, Taipei, Taiwan; ${ }^{4}$ Engelhardt Institute of Molecular Biology, Russian Academy of Sciences, Moscow, Russia ${ }^{5}$ Moscow Research and Clinical Center for Tuberculosis Control, Moscow, Russia

Second-line injection drugs group, which include kanamycin (KAN), capreomycin (CAP), and amikacin (AM), is one of the cornerstones used in the treatment of MDR tuberculosis. Though the main resistance mechanism leading to cross-resistance to all three drugs described in Mycobacterium tuberculosis is the alteration of $16 \mathrm{~S}$ rRNA, other mechanisms also could be found in clinical strains, such as promoter mutations of the eis and whiB 7 genes leading to KAN resistance, and TlyA inactivating mutations leading to CAP resistance. In consequence, a noticeable number of resistant strains do not carry any known mutations.

We performed the next-generation sequence analysis of the 5 Beijing and one Haarlem lineage clinical $M$. tuberculosis strains with discordant results of phenotypic resistance to injection drugs, and genetic analysis of $r r s$, eis, tly $A$, and whiB7loci. The sequencing data were analyzed using the Galaxy web platform (http://usegalaxy.org). Further bioinformatic analysis of the obtained SNPs was performed with custom Python scripts and public databases ReSeqTB and PolyTB.

We found 2126-2229 SNPs for Beijing lineage and 1606 SNPs for Haarlem lineage isolates compared to the referent H37 Rv strain. Upon the exclusion of known mutations associated with resistance, fitness compensation and deep bioinformatic analysis, the list of candidate SNPs, potentially associated with resistance, was shortened to $10-100$ for each strain. The novel putative mechanisms of resistance included mutations in elongation factor EF-G, phosphotransferase Aph, hypothetical protein Rv0147, secretion protein EspG2, and aspartate aminotransferase AspC.

The diversity of drug resistance mechanisms reflects the complexity of microevolution of $M$. tuberculosis and impacts the sensitivity of molecular tests. Improvement of our knowledge of drug-resistance mechanisms would facilitate the discovery of new drugs together with the prediction of drugs interactions and promote the development of molecular assays.

This study was funded by the Russian-Taiwanese Joint Research Project supported by the Ministry of Science and Technology, Taiwan (MOST) grant number MOST 105-2923-B-404-001MY3 and Russian Foundation for Basic Research (RFBR), grant \#16-54-52043. 